\title{
Reduced Order Modeling of Time-Dependent Reflectance Profiles from Purely Scattering Media
}

\author{
R. Scott Larson \\ Brigham Young University, slrussia@gmail.com \\ Matthew R. Jones \\ Brigham Young University - Provo, mrjones@byu.edu
}

Follow this and additional works at: https://scholarsarchive.byu.edu/facpub

Part of the Mechanical Engineering Commons

\section{Original Publication Citation}

Journal of Quantitative Spectroscopy \& Radiative Transfer 109 (2008) 201-209

\section{BYU ScholarsArchive Citation}

Larson, R. Scott and Jones, Matthew R., "Reduced Order Modeling of Time-Dependent Reflectance Profiles from Purely Scattering Media" (2008). Faculty Publications. 3187.

https://scholarsarchive.byu.edu/facpub/3187 


\title{
Reduced-order modeling of time-dependent reflectance profiles from purely scattering media
}

\author{
R. Scott Larson, Matthew R. Jones* \\ Department of Mechanical Engineering, Brigham Young University, Provo, UT 84602, USA \\ Received 15 February 2007; accepted 29 August 2007
}

\begin{abstract}
Due to the widespread existence and importance of foam, inverse techniques for characterizing industrial foams are of interest. An essential element in an inverse method used to characterize a foam layer is a model of the time-dependent reflectance of a laser pulse. Monte Carlo methods may be used to accurately model reflectance, but these methods are computationally expensive. Computationally efficient methods based on the diffusion approximation have been developed, but this approach is not sufficiently accurate in many cases of interest. Therefore, a computationally efficient and robust method is desirable. This paper presents a computationally efficient method for modeling the time-dependent reflectance of a laser pulse from a non-absorbing, scattering plane layer that is based on reduced-order modeling techniques. The accuracy of the proposed method is demonstrated by comparing reflectance profiles for randomly selected foam layer properties with corresponding profiles that were generated from Monte Carlo simulations.
\end{abstract}

(C) 2007 Elsevier Ltd. All rights reserved.

Keywords: Transient radiative transfer; Reduced-order modeling; Monte Carlo simulations

\section{Introduction}

The formation of foam is a familiar phenomenon. It is seen in beverages, shaving cream, soaps and other cleaning agents. Its properties are exploited in advanced oil recovery, fire fighting, insulation products and the production of chemicals. Foam is formed in many industrial processes. In many cases, this formation negatively impacts the transport of heat and mass occurring during the process. In industrial glass furnaces the formation of foam on the batch melt is very undesirable and costly. In a typical glassmelt about one-third of the surface of the molten glass is covered by foam of varying thicknesses [1]. It has been estimated that as much as $60 \%$ of the radiative heat flux is either blocked or scattered by this foam layer [2]. This insulating effect results in a significant reduction in energy efficiency of the furnace. To overcome the additional resistance to heat transfer created by a foam layer, glass-melting furnaces are operated at elevated temperatures. In addition to increasing the amount of fuel required, higher temperature operation leads to an increase in $\mathrm{NO}_{x}$ gas emissions.

\footnotetext{
*Corresponding author. Tel.: + 18014223070 ; fax: + 18014220516.

E-mail address: mrjones@byu.edu (M.R. Jones).
} 


\section{Nomenclature}

a column of reflectance profile

A matrix of reflectance profiles

b vector of expansion coefficients

B matrix of expansion coefficients

C matrix of expansion coefficients

$D_{j} \quad$ number of photons detected

f column vector of interpolation functions

F matrix of interpolation functions

$\mathbf{F}^{+} \quad$ Moore-Penrose pseudoinverse

$g \quad$ asymmetry parameter

$\mathbf{k} \quad$ vector of parameters

$L \quad$ layer thickness

$m \quad$ number of reflectance profiles

$M \quad$ number of columns in truncated basis

$n \quad$ number of time steps

$P \quad$ number of photons leaving laser

$r_{\mathrm{a}} \quad$ rank of matrix $\mathbf{A}$

$r_{\mathrm{d}} \quad$ radial location of detector

$r_{\mathrm{o}} \quad$ radius of laser

$\mathbf{R}$ matrix of reflectance profiles

$R_{j} \quad j$ th element of reflectance time profile

$R_{\mathrm{MC}, i} \quad$ reflectance predicted by $\mathrm{MC}$

$R_{\mathrm{ROM}, i}$ reflectance predicted by ROM

U SVD component

V SVD component

$\delta_{i} \quad$ difference between ROM and MC

$\Delta r \quad$ radial resolution

$\mu_{\mathrm{s}} \quad$ scattering coefficient

$\sigma \quad$ singular value

$\Sigma \quad$ SVD component whose diagonal elements are singular values

Ф matrix of truncated basis vectors

In order to evaluate the radiative heat transfer in glass foam formation its radiative properties need to be known. Methods for determining the radiative properties of glass foams are reviewed extensively by Randrianalisoa et al. [3].

A spectroscopic method of measuring the properties of foam in a glass furnace has been proposed [4]. In this method, a pulsed laser beam is used to illuminate the foam layer. The laser radiation is absorbed and scattered as it travels through the foam layer. A portion of the radiation passes through the foam and into the glass melt. Some of the radiation is reflected out of the foam (see Fig. 1). This reflected radiation is measured at different radial locations away from the laser. The measured time-dependent reflectance profiles are input to an inverse method in order to estimate the properties of the foam. Because the inverse method requires multiple solutions of the forward model of the radiation process, the objective of this paper is to demonstrate an efficient and accurate model of the radiative transport in the foam layer.

Propagation of the laser beam through a non-absorbing foam layer has been modeled using a Monte Carlo (MC) approach [4]. However, use of MC simulations to solve the inverse problem is infeasible due to the computational expense. Depending on the properties of the foam layer (thickness, scattering coefficient and asymmetry parameter), a MC simulation requires between 20 and $200 \mathrm{~h}$ on a SGI Origin supercomputer with 64 MIPS processors. Computationally efficient approximate methods based on the diffusion approximation 


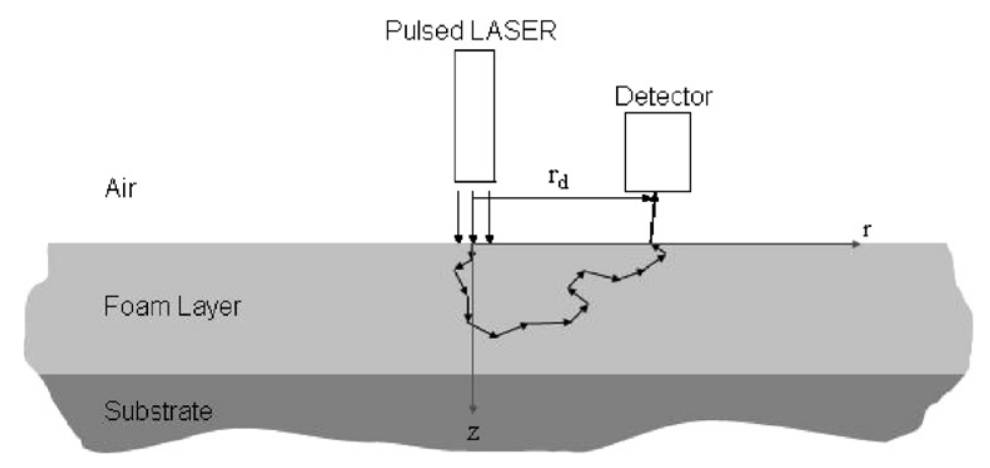

Fig. 1. Illustration of the spectroscopic method.

have been explored, but these methods are not sufficiently accurate in all cases of interest [4]. Therefore, the need for a robust, computationally efficient method for predicting the time-dependent reflectance profiles exits.

A possible approach is the use of reduced order or spectral methods that obtain a low-dimensional approximate description of a high-dimensional process. The basic idea underlying each of the reduced-order modeling techniques presented in the literature is to use the singular value decomposition (SVD) (or its mathematical equivalent) of a matrix containing representative data to generate an orthonormal basis for the process to be modeled. These methods have been used in signal processing, pattern recognition, control theory, fluid flow and dynamics for about a century [5-8]. Recently, this approach has been used in an inverse method, which is designed to estimate thermal conductivities and convection heat transfer coefficients [9]. Since the reduced-order method used in this study has been 'discovered' by numerous people working on a wide variety of problems, there exits a confusing array of names and acronyms for the approach described here [10]. It is frequently referred to as proper orthogonal decomposition, principal component analysis or as the Karhunen-Loeve decomposition [5-9].

The results presented in this paper demonstrate that reduced-order models can be used to accurately predict the time-dependent reflectance profiles when a laser beam is incident on a non-absorbing, scattering plane layer with arbitrary thickness, scattering coefficient and asymmetry parameter. It is anticipated that this development will lead to an inverse method capable of accurately characterizing the radiative and structural properties of foam layers that form in a wide variety of industrial processes.

\section{Monte Carlo modeling}

MC simulations are often used as benchmarks to which other methods of modeling radiative transfer are compared [11]. The MC algorithm used in this study was adapted from the code written by Salisbury [4], which was based on the method outlined by Jacques and Wang [12]. This code simulates the random path that a photon may take as it traverses through the medium. The propagation of photons is based on probability distributions which are functions of the set of governing parameters, $\mathbf{k}$. These probability distributions are used to calculate the path length between scattering events and the deflection angles resulting from a scattering event.

As was illustrated in Fig. 1, photons are normally incident on a plane layer. A small fraction of the photon paths will eventually intersect the detector, and the number of photons incident on the detector within the time window $t_{j}+\Delta t_{j}$ is recorded as $D_{j}$. The $j$ th element of an $n$-dimensional vector representing the time-dependent reflectance is given by

$$
R_{j}=\frac{D_{j}}{P} \frac{r_{\mathrm{o}}^{2}}{2 r_{\mathrm{d}} \Delta r}
$$

In each of the MC simulations presented in this paper, the number of photons released into the medium, $P$, is equal to $10^{7}$. The spacing between the detector and the laser $r_{\mathrm{d}}$ is $5 \mathrm{~mm}$, the radius of the laser $r_{\mathrm{o}}$ is $1 \mathrm{~mm}$ and 
the radial resolution $\Delta r$ is $1 \mathrm{~mm}$. Each simulation was repeated 10 times, and the uncertainty for each reflectance profile was calculated using finite statistical theory with a 95\% confidence interval. Complete details regarding the uncertainty estimates are given by Salisbury [4].

\section{Reduced-order modeling}

The implementation of reduced-order modeling is relatively simple once the parameters governing the phenomenon of interest are identified. Data sets describing the phenomenon at specified values of the governing parameters are obtained by experiment or by analysis [9]. The data sets are arranged in a matrix and an orthonormal basis for the column space of this matrix is obtained from its SVD. The basis vectors are ordered according to the magnitude of their associated characteristic values, and the first few basis vectors contain the majority of the information required to describe the phenomenon. Therefore, a truncated set of the basis vectors can be used to estimate the phenomenon of interest for an arbitrary set of the governing parameters. The expansion coefficients, which are functions of the set of governing parameters, are determined by curve fitting the original data set.

The following paragraphs describe this method in more detail while focusing on the application of interest in this paper, which predicts the time-dependent reflectance profile produced when a pulsed laser beam is normally incident on a non-absorbing, scattering plane layer. The properties of the layer, which form the set of governing parameters, are the scattering coefficient, the asymmetry parameter and the layer thickness.

$$
\mathbf{k}=\left[\mu_{\mathrm{s}}, g, L\right]^{\mathrm{T}} .
$$

The fundamental concept underlying the reduced-order model is that the reflectance profile for an arbitrary parameter set can be approximated by a linear combination of a set of orthonormal basis vectors.

$$
\mathbf{R}_{\mathbf{k}} \approx \boldsymbol{\Phi b}(\mathbf{k}) .
$$

In this equation, $\boldsymbol{\Phi}$ represents the basis vectors, $\mathbf{b}$ represents the expansion coefficients, which are functions of the set of governing parameters. The major tasks required in the development of the reduced-order model are the identification of the basis vectors and the functional relationship between the expansion coefficients and the set of governing parameters.

\subsection{Calculation of the truncated set of basis vectors}

In the present study, MC simulations were used to generate reflectance profiles for each possible combination of the following values of the governing parameters: $\mu_{\mathrm{s}}=(2800,5600,8400,11,200,14,000) \mathrm{m}^{-1}$, $g=(0.2,0.4,0.6,0.8)$ and $L=(0.04,0.08,0.12,0.16,0.2) \mathrm{m}$. The detector was located at a distance of $r_{\mathrm{d}}=5 \mathrm{~mm}$ from the centerline of the laser beam, and the reflected flux was measured as a function of time for $16 \mathrm{~ns}$ with a time resolution of $20 \mathrm{ps}$. Therefore, this process resulted in $m=100$ reflectance profiles, each of which consists of $n=800$ time-dependent reflectance measurements. Each reflectance profile forms a column in an $n \times m$ matrix $\mathbf{A}$.

Using the SVD, A can be factored into an $n$ by $n$ orthogonal matrix $\mathbf{U}$, an $n$ by $m$ diagonal matrix $\boldsymbol{\Sigma}$ and an $m$ by $m$ orthogonal matrix $\mathbf{V}$ as shown in Eq. (4) [13]:

$$
\mathbf{A}=\mathbf{U}_{\mathrm{A}} \boldsymbol{\Sigma}_{\mathrm{A}} \mathbf{V}_{\mathrm{A}}^{\mathbf{T}}
$$

The elements of $\boldsymbol{\Sigma}_{\mathrm{A}}$ are termed the singular values. The number of non-zero singular values is equal to the rank of $\mathbf{A}$ and is given the symbol $r_{\mathrm{A}}$. The singular values are represented by $\sigma_{\mathrm{A} i}, i=1, \ldots, r_{\mathrm{A}}[13]$.

The first $r_{\mathrm{A}}$ columns of $\mathbf{U}_{\mathrm{A}}$ form an orthonormal basis for the column space of $\mathbf{A}$. However, it is unnecessary to use the entire basis to accurately represent the reflectance profiles, so only the first $M$ columns of $\mathbf{U}_{\mathrm{A}}$ are used to produce the truncated set of basis vectors, $\boldsymbol{\Phi}$, required by Eq. (3). As shown in Fig. 2, the singular values of A decrease rapidly. For this study, based on Fig. 2, $M$ is set equal to 6. It will be shown that the basis 


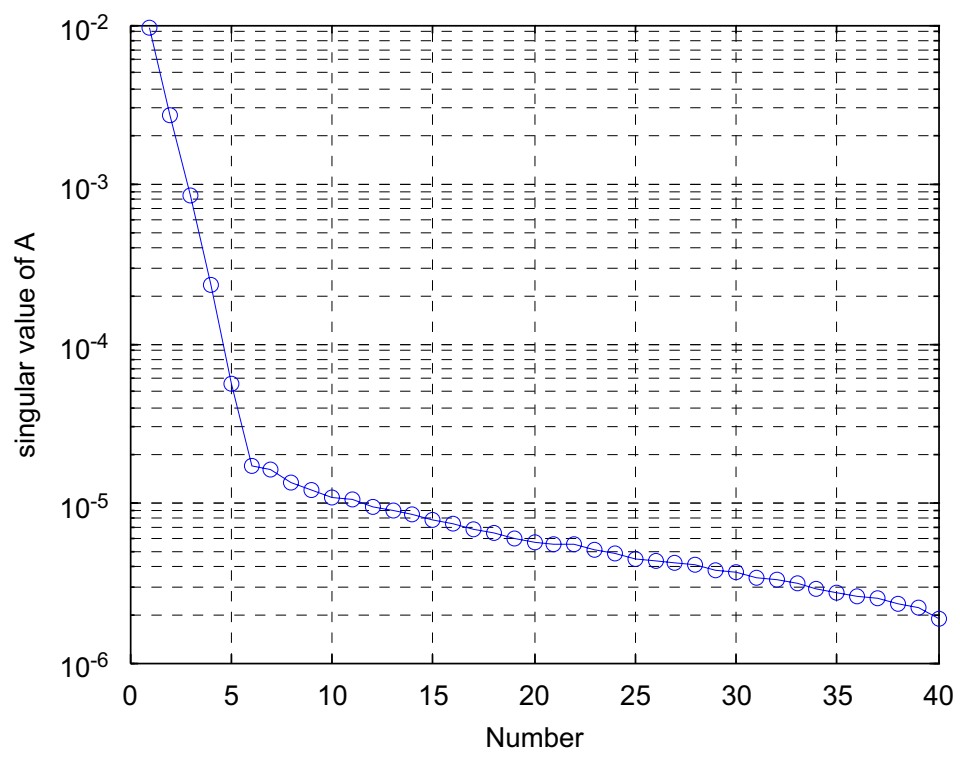

Fig. 2. Singular values of $\mathbf{A}$.

vectors corresponding to the six largest singular values contain sufficient information to reconstruct an arbitrary reflectance profile. Including more vectors than this does not significantly improve the accuracy of the solution.

\subsection{Calculation of the expansion coefficients}

The $i$ th column of $\mathbf{A}, \mathbf{a}_{i}$, represents the time-dependent reflectance profile obtained when the layer has properties corresponding to the $i$ th set of governing parameters. This reflectance profile can now be approximated using the truncated set of basis vectors as shown in Eq. (5):

$$
\mathbf{a}_{i} \approx \boldsymbol{\Phi b}\left(\mathbf{k}^{i}\right) .
$$

Eq. (5) can be rewritten in matrix form as

$$
\mathbf{A}=\mathbf{\Phi B},
$$

where $\mathbf{B}$ is an $M$ by $m$ matrix whose $i$ th column contains the expansion coefficients required to reconstruct the reflectance profile corresponding to the $i$ th parameter set. Because of the orthogonality of $\boldsymbol{\Phi}, \mathbf{B}$ is readily calculated as shown in Eq. (7):

$$
\mathbf{B}=\boldsymbol{\Phi}^{\mathrm{T}} \mathbf{A} .
$$

Eq. (7) gives the expansion coefficients for each of the specified parameter sets used to create $\mathbf{A}$. The ability to calculate the expansion coefficients for an arbitrary parameter set, $\mathbf{b}(\mathbf{k})$, is required. Estimates of expansion coefficients may be obtained by interpolation of the results given by Eq. (7). This is done by defining a coefficient matrix $\mathbf{C}$ such that

$$
\mathbf{B}=\mathbf{C F},
$$

where $\mathbf{F}$ is an $m$ by $m$ matrix of interpolating functions. While any interpolating functions may be used, previous studies have found that inverse multiquadric functions are useful when interpolating data in more 
than one dimension [14,15]. The $i$ th column of $\mathbf{F}$ is calculated by evaluating the multiquadric interpolation function defined in Eq. (9) for each of the parameter sets used to generate $\mathbf{A}$.

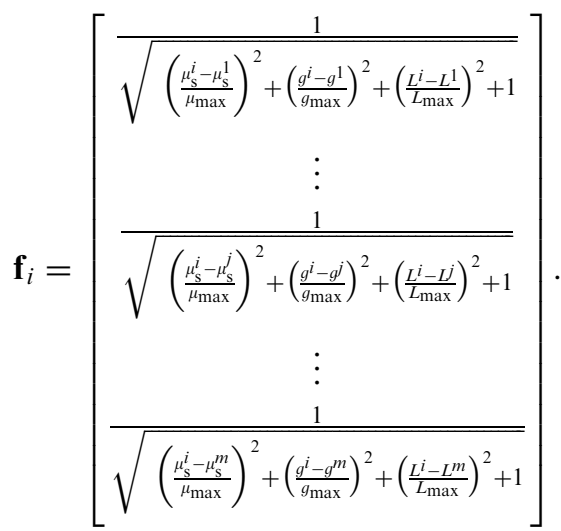

Since $\mathbf{F}$ is a singular matrix, Eq. (8) must be solved for the coefficient matrix $\mathbf{C}$ in a least-squares sense using the Moore-Penrose pseudoinverse, $\mathbf{F}^{+}[13,16]$. Calculation of the pseudoinverse is also based on the SVD, but this time, the interpolation matrix is factored.

$$
\mathbf{F}=\mathbf{U}_{\mathrm{F}} \boldsymbol{\Sigma}_{\mathrm{F}} \mathbf{V}_{\mathrm{F}}^{\mathrm{T}}
$$

$\mathbf{U}_{\mathrm{F}}$ and $\mathbf{V}_{\mathrm{F}}$ are orthonormal matrices and $\boldsymbol{\Sigma}_{\mathrm{F}}$ is a diagonal matrix containing the singular values of $\mathbf{F}$, $\left(\sigma_{\mathrm{F} i}, i=1, \ldots, r_{\mathrm{F}}\right)$. The pseudoinverse is calculated as shown in Eq. (11):

$$
\mathbf{F} \mathbf{V}_{\mathrm{F}} \mathbf{S}_{\mathrm{F}} \mathbf{U}_{\mathrm{F}}^{\mathrm{T}}=\mathbf{U}_{\mathrm{F}} \Sigma_{\mathrm{F}} \mathbf{V}_{\mathrm{F}}^{\mathrm{T}} \mathbf{V}_{\mathrm{F}} \mathbf{S}_{\mathrm{F}} \mathbf{U}_{\mathrm{F}}^{\mathrm{T}}=\mathbf{I},
$$

where $\mathbf{S}_{\mathrm{F}}$ is a diagonal matrix that is defined as

$$
\mathbf{S}_{\mathrm{F}}=\operatorname{diag}\left\{1 / \sigma_{\mathrm{F}, i}\right\},
$$

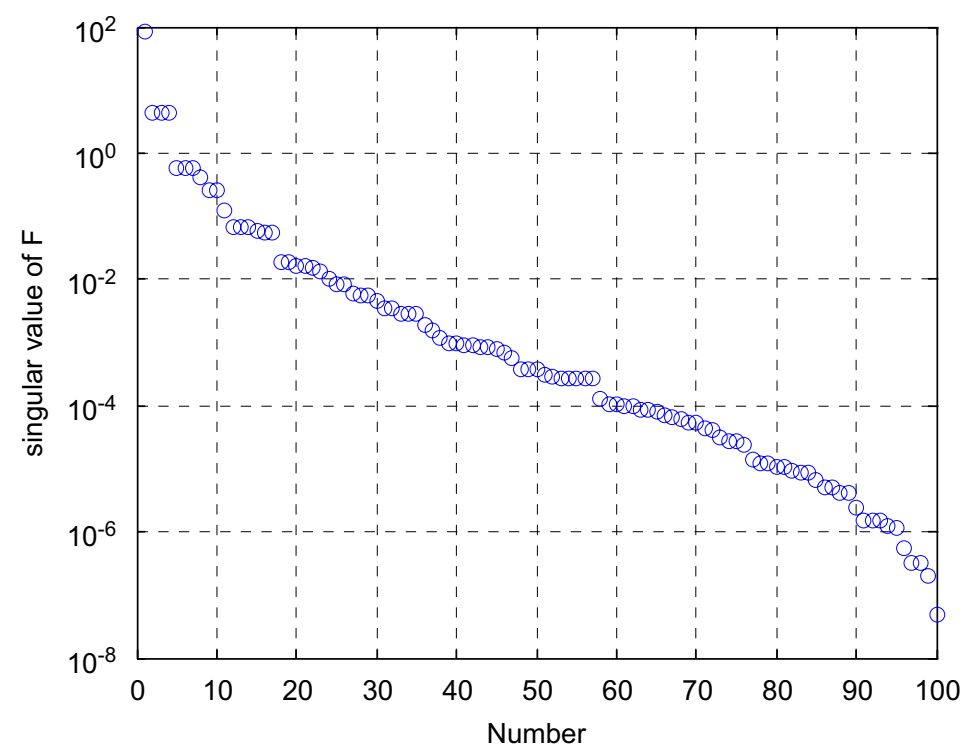

Fig. 3. Singular values of $\mathbf{F}$. 
for $\sigma_{\mathrm{F}, i}>0$. In practice, it is necessary to specify a minimum allowable value for $\sigma_{\mathrm{F}, i}$ and to set the elements of $\mathbf{S}_{\mathrm{F}}$ equal to zero if $\sigma_{\mathrm{F}, i}$ is less than this specified minimum value. The singular values of the interpolation matrix used in this study are shown in Fig. 3. The results presented in the following section were obtained with a minimum allowable singular value of $10^{-4}$.

From Eq. (11), it is clear that the pseduoinverse of $\mathbf{F}$ is given by

$$
\mathbf{F}^{+}=\mathbf{V}_{\mathrm{F}} \mathbf{S}_{\mathrm{F}} \mathbf{U}_{\mathrm{F}}^{\mathrm{T}} \text {. }
$$

Once the pseudoinverse of $\mathbf{F}$ is known, the coefficient matrix is obtained by post-multiplying Eq. (8) by $\mathbf{F}^{+}$.

$$
\mathbf{C}=\mathbf{B F}^{+} \text {. }
$$

It is assumed that this coefficient matrix is valid for any arbitrary parameter set. Therefore, expansion coefficients for any arbitrary parameter set may be obtained by evaluating the interpolation function at $\mathbf{k}$, and

a

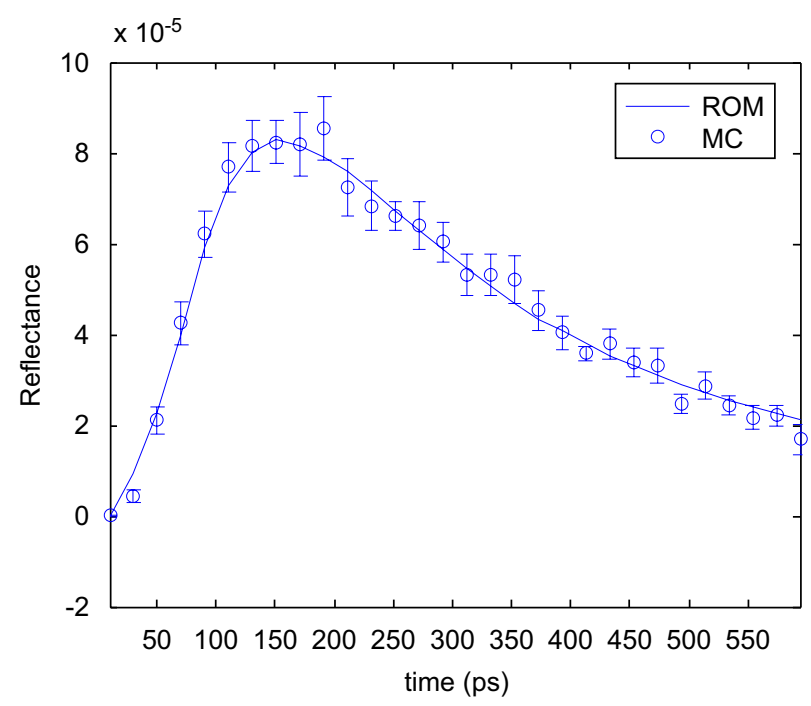

b

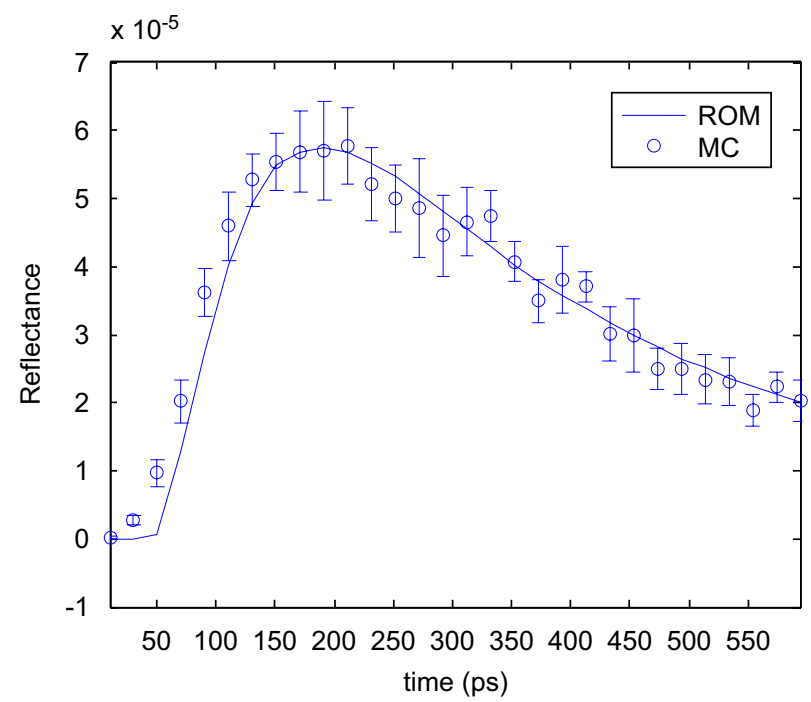

C

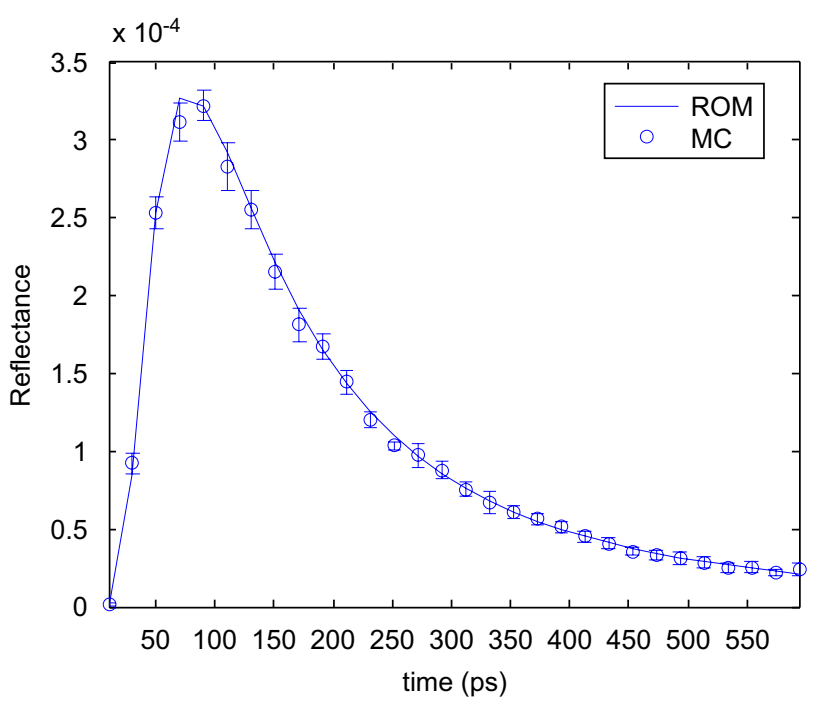

d

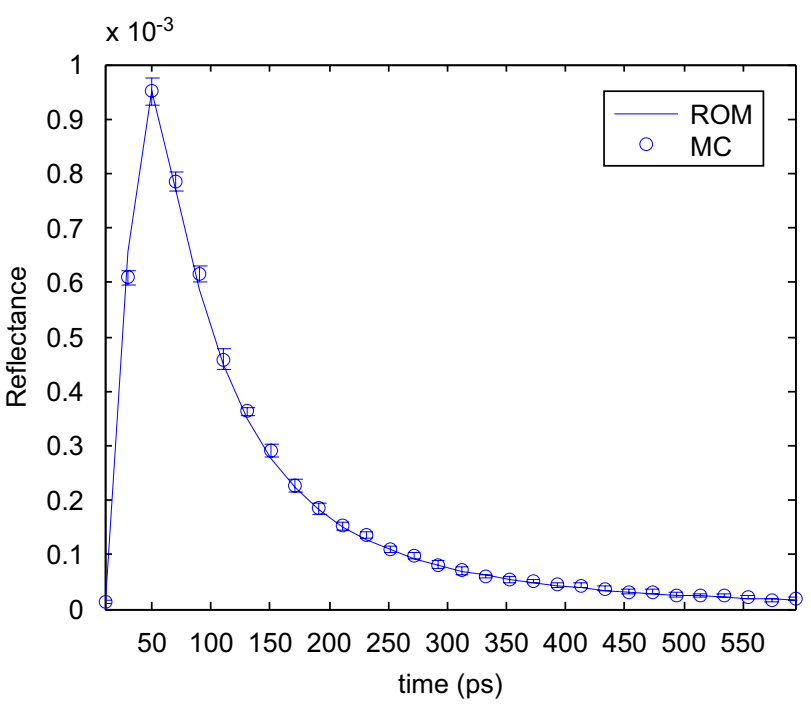

Fig. 4. Comparison of reflectance profiles obtained using ROM with the corresponding MC simulation for (a) case 1 with parameters $\mu_{\mathrm{s}}=11,620 \mathrm{~m}^{-1}, g=0.5154, L=0.1415 \mathrm{~m}$, (b) case 2 with parameters $\mu_{\mathrm{s}}=13,760 \mathrm{~m}^{-1}, g=0.5066, L=0.1963 \mathrm{~m}$, (c) case 4 with parameters $\mu_{\mathrm{s}}=12,760 \mathrm{~m}^{-1}, g=0.7888, L=0.0989 \mathrm{~m}$ and (d) case 6 with parameters $\mu_{\mathrm{s}}=3768 \mathrm{~m}^{-1}, g=0.6710, L=0.1690 \mathrm{~m}$. 
Table 1

Summary of the results for randomly generated test cases

\begin{tabular}{|c|c|c|c|c|c|}
\hline Case & $g$ & $L(\mathrm{~m})$ & $\mu_{\mathrm{s}}\left(\mathrm{m}^{-1}\right)$ & $\delta_{\mathrm{A}}$ & $R^{2}$ \\
\hline 1 & 0.5154 & 0.1415 & 11,620 & 0.0497 & 0.9834 \\
\hline 2 & 0.5066 & 0.1963 & 13,760 & 0.0754 & 0.9500 \\
\hline 3 & 0.4330 & 0.0500 & 7840 & 0.0620 & 0.9685 \\
\hline 4 & 0.7888 & 0.0989 & 12,760 & 0.0250 & 0.9976 \\
\hline 5 & 0.8964 & 0.0403 & 3173 & 0.2860 & 0.9246 \\
\hline 6 & 0.6710 & 0.1690 & 3768 & 0.0319 & 0.9976 \\
\hline 7 & 0.6590 & 0.1800 & 13,410 & 0.0698 & 0.9651 \\
\hline 8 & 0.2970 & 0.0530 & 4950 & 0.0523 & 0.9844 \\
\hline 9 & 0.6920 & 0.1260 & 9865 & 0.0587 & 0.9763 \\
\hline 10 & 0.5390 & 0.1540 & 9737 & 0.0520 & 0.9775 \\
\hline
\end{tabular}

post-multiplying $\mathbf{C}$ with the resulting vector

$$
\mathbf{b}(\mathbf{k})=\mathbf{C f}(\mathbf{k}) \text {, }
$$

where

$$
\mathbf{f}(\mathbf{k})=\left[\begin{array}{c}
\frac{1}{\sqrt{\left(\frac{\mu_{\mathrm{s}}-\mu_{\mathrm{s}}^{1}}{\mu_{\max }}\right)^{2}+\left(\frac{g-g^{1}}{g_{\max }}\right)^{2}+\left(\frac{L-L^{1}}{L_{\max }}\right)^{2}+1}} \\
\vdots \\
\frac{1}{\sqrt{\left(\frac{\mu_{\mathrm{s}}-\mu_{\mathrm{s}}^{\mathrm{s}}}{\mu_{\max }}\right)^{2}+\left(\frac{g-g^{j}}{g_{\max }}\right)^{2}+\left(\frac{L-L^{j}}{L_{\max }}\right)^{2}+1}} \\
\vdots \\
\frac{1}{\sqrt{\left(\frac{\mu_{s}-\mu_{s}^{m}}{\mu_{\max }}\right)^{2}+\left(\frac{g-g^{m}}{g_{\max }}\right)^{2}+\left(\frac{L-L^{m}}{L_{\max }}\right)^{2}+1}}
\end{array}\right] .
$$

The expansion coefficients given by Eq. (15) are then used in Eq. (3) to calculate the reflectance profile for any arbitrary parameter set, $\mathbf{k}$.

\section{Results}

Reflectance profiles were calculated using the reduced-order model for 10 randomly selected parameter sets lying with the parameter ranges used to generate the reduced-order model. These reflectance profiles were compared with the corresponding MC simulations, and representative results are shown in Fig. 4.

The results for all 10 cases are summarized in Table 1. The difference between the MC and ROM reflectance profiles is quantified as the difference in the areas under the respective curves normalized by the area under the MC curve.

$$
\delta_{i}=\left|\frac{A_{\mathrm{ROM}, i}-A_{\mathrm{MC}, i}}{A_{\mathrm{MC}, i}}\right|,
$$

Also, because the reduced-order model is essentially a curve fit of the MC data, an $R^{2}$ value is calculated following standard practice [17].

\section{Summary and conclusions}

The use of a reduced-order model to predict the time-dependent reflectance of a laser pulse from a nonabsorbing, scattering plane layer has been investigated. Comparison of reflectance profiles obtained using the 
reduced-order model with corresponding Monte Carlo simulations shows that the proposed approach is highly accurate.

Once the data for the library were generated and reduced to a set of basis functions and an interpolating function, the time-dependent reflectance profiles were obtained essentially instantaneously using a reducedorder model run on a desktop PC. Generation of the corresponding reflectance profile by executing a MC simulation on a supercomputer would require anywhere between 20 and $200 \mathrm{~h}$. It is anticipated that the computational efficiency of reduced-order models will lead to an increased ability to solve inverse radiative transfer problems, and the implementation of reduced-order modeling in inverse algorithms is currently being investigated.

Therefore, it is concluded that reduced-order modeling techniques are a powerful tool for accurately and efficiently modeling a transient radiative transfer process once the library is in place. Creation of the library may be computationally expensive, but the results of this study show that a library based on course resolution of the governing parameters gives highly satisfactory results. The library of 100 solutions used in this study was based on the combination of three parameters - two with five values and one with four values.

The results also show that it is important to accurately estimate the range of the governing parameters and to ensure that the library includes all possible values for these parameters. Extrapolation outside of the library range is ill advised as can be seen from the relatively poor results obtained for case 5 . The asymmetry parameter used in that case was 0.8964 , and the upper limit used to create the library was 0.800 . Also, as can be seen in case 2, the error is increased as the value of the parameters near the upper and lower limits of the parameter range. Case 2 has a scattering coefficient of $13,760 \mathrm{~m}^{-1}$, which is near the bound of $14,000 \mathrm{~m}^{-1}$, and the results obtained in this case are also relatively poor.

\section{References}

[1] Fedorov AG, Viskanta R. Radiation characteristics of glass foams. J Am Ceram Soc 2000;83(11):2769-76.

[2] Trier W. Wärmeübergang zwischen flamme and glasbad in glasschmelxwannenöfen. Glastech Ber 1963;36(3):73-86.

[3] Randrianalisoa J, Baillis D, Pilon L. Improved inverse method for radiative characteristics of closed-cell absorbing porous media. J Thermophys Heat Transfer 2006;20(4):871-83.

[4] Salisbury SM. A method for characterizing the properties of industrial foams. Masters thesis, Brigham Young University, 2005.

[5] Pearson K. On lines planes of closest fit to system of points in space. London Edinb Dublin Philos Mag J Sci 1901;2:559-72.

[6] Hoteling H. Analysis of complex statistical variables into principal components. J Educ Psychol 1983;24:417-41.

[7] Atwell JA, King BB. Proper orthogonal decomposition for reduced basis feedback controllers for parabolic equations. Math Comput Modeling 2001;33:1-19.

[8] Berkooz G, Holmes P, Lumley JL. The proper orthogonal decomposition in the analysis of turbulent flows. Annu Rev Fluid Mech 1993;25(5):539-75.

[9] Ostrowski Z, Bialecki RA, Kassab AJ. Estimation of constant thermal conductivity by use of proper orthogonal decomposition. Comput Mech 2005;37:52-9.

[10] Humphreys J. Personal communication, Department of Mathematics, Brigham Young University.

[11] Flock ST, Patterson MS, Wilson BC, Wyman DR. Monte Carlo modeling of light propogation in highly scattering tissues-I: model predictions and comparison with diffusion theory. IEEE Trans Biomed Eng 1989;36(12):1162-7.

[12] Jacques SL, Wang L, Hielscher AH. Time-resolved photon propagation in tissues. In: Welch AJ, van Gemert MJC, editors. Optical-thermal response of laser-irradiated tissue. New York: Plenum Press; 1995. p. 305-32.

[13] Strang G. Linear algebra and its application. Belmont, CA: Thomson, Brooks/Cole; 2006.

[14] Hardy RL. Multiquadric equations of topography and other irregular surfaces. J Geophys Res 1971;76:1905-15.

[15] Hardy RL. Theory and applications of the multiquadric-biharmonic method: 20 years of discovery 1968-1988. Comput Math Appl 1990;19:163-208.

[16] Press WH, Teukolsky SA, Vetterling WT, Flannery BP. Numerical recipes in FORTRAN: the art of scientific computing. Cambridge: Cambridge University Press; 1992.

[17] Lawson J, Erjavec J. Modern statistics for engineering and quality improvement, Wadsworth Group, 2001. 